\title{
Traversable wormholes in Einstein-Dirac-Maxwell theory
}

\author{
Jose Luis Blázquez-Salcedo, ${ }^{1,2}$ Christian Knoll, ${ }^{2}$ and Eugen Radu ${ }^{3}$ \\ ${ }^{1}$ Departamento de Física Teórica and IPARCOS, \\ Universidad Complutense de Madrid, E-28040 Madrid, Spain \\ ${ }^{2}$ Institut für Physik, Universität Oldenburg, Postfach 2503, D-26111 Oldenburg, Germany \\ ${ }^{3}$ Departamento de Matemática da Universidade de Aveiro and \\ Centre for Research and Development in Mathematics and Applications (CIDMA), \\ Campus de Santiago, 3810-183 Aveiro, Portugal
}

(Dated: March 15, 2021)

\begin{abstract}
We construct a specific example of a class of traversable wormholes in Einstein-Dirac-Maxwell theory in four spacetime dimensions, without needing any form of exotic matter. Restricting to a model with two massive fermions in a singlet spinor state, we show the existence of spherically symmetric asymptotically flat configurations which are free of singularities, representing localized states. These solutions satisfy a generalized Smarr relation, being connected with the extremal Reissner-Nordström black holes. They also possess a finite mass $M$ and electric charge $Q_{e}$, with $Q_{e} / M>1$. An exact wormhole solution with ungauged, massless fermions is also reported.
\end{abstract}

Introduction.- The wormholes (WHs) have entered modern physics soon after the discovery of black holes (BHs) [1, 2]. In both cases it took decades to understand their rich physical content and to realize that they may play a role in Nature. However, while there is increasing evidence for the existence of (astrophysical) BHs, the (Lorentzian, traversable) WHs remain so far rather an interesting possibility, although with observational implications [3]. A basic difference between these two types of solutions occurs already at the level of energy-momentum supporting the corresponding geometries. While the BHs exist in vacuum, being the end point of (normal matter's) gravitational collapse, the traversable WHs necessarily require a matter content violating the null energy condition [4], [5]. Restricting to a field theory source and a classical setting, the (bosonic) matter fields necessarily possess a non-standard Lagrangian (e.g. 'phantom' fields [6]), or one has to consider extensions of gravity beyond general relativity (see e.g. 7], [8]).

However, as we shall prove in this work, the situation changes for fermions, with the existence of traversable WHs solutions of the Einstein-Dirac equations. In our approach, the Dirac matter is described by a quantum wave function rather than a quantum field. This results in a more tractable model, with the backreaction of the matter to spacetime geometry being taken into account. Moreover, the inclusion of an electric charge leads to 'smooth' geometries, without the presence of a thin shell of extra-matter at the throat of the WH.

Einstein-Dirac-Maxwell model.- We consider a model with two gauged relativistic fermions, the spin of which is taken to be opposite in order to satisfy spherical symmetry. Working in units with $G=c=$ $\hbar=1$, the action of the corresponding Einstein-Dirac-
Maxwell (EDM) model reads

$$
S=\frac{1}{4 \pi} \int \mathrm{d}^{4} x \sqrt{-g}\left[\frac{1}{4} R+\mathcal{L}_{D}-\frac{1}{4} F^{2}\right],
$$

where $R$ is the Ricci scalar of the metric $g_{\mu \nu}, F_{\mu \nu}=$ $\partial_{\mu} A_{\nu}-\partial_{\nu} A_{\mu}$ is the field strength tensor of the $\mathrm{U}(1)$ field $A_{\mu}$, and

$$
\mathcal{L}_{D}=\sum_{\epsilon=1,2}\left[\frac{\mathrm{i}}{2} \bar{\Psi}_{\epsilon} \gamma^{\nu} \hat{D}_{\nu} \Psi_{\epsilon}-\frac{\mathrm{i}}{2} \hat{D}_{\nu} \bar{\Psi}_{\epsilon} \gamma^{\nu} \Psi_{\epsilon}-\mu \bar{\Psi}_{\epsilon} \Psi_{\epsilon}\right],
$$

where $\gamma^{\nu}$ are the curved space gamma matrices [9] and $\mu$ is the mass of both spinors $\Psi_{\epsilon=1,2}$. Also, $\hat{D}_{\mu}=$ $\partial_{\mu}+\Gamma_{\mu}-i q A_{\mu}$, where $\Gamma_{\mu}$ are the spinor connection matrices, and $q$ is the gauge coupling constant. The resulting field equations are

$$
\begin{aligned}
& R_{\mu \nu}-\frac{1}{2} R g_{\mu \nu}=2 T_{\mu \nu} \text { with } T_{\mu \nu}=T_{\mu \nu}^{(D)}+T_{\mu \nu}^{(M)}, \\
& \left(\gamma^{\nu} \hat{D}_{\nu}-\mu\right) \Psi_{\epsilon}=0, \quad \nabla_{\mu} F^{\mu \nu}=q j^{\nu}
\end{aligned}
$$

with the current $j^{\nu}=\sum_{\epsilon=1,2} \bar{\Psi}_{\epsilon} \gamma^{\nu} \Psi_{\epsilon}$ and $T_{\mu \nu}^{(D)}=$ $\sum_{\epsilon=1,2} 2 \Im\left(\bar{\Psi}_{\epsilon} \gamma_{(\mu} \hat{D}_{\nu)} \Psi_{\epsilon}\right), T_{\mu \nu}^{(M)}=F_{\mu \alpha} F_{\nu}^{\alpha}-\frac{1}{4} F^{2} g_{\mu \nu}$.

Restricting to static, spherically-symmetric solutions of the field equations, we consider a general metric ansatz $d s^{2}=g_{t t}(r) d t^{2}+g_{r r}(r) d r^{2}+g_{\Omega \Omega}(r) d \Omega^{2}$, where $r$ and $t$ are the radial and time coordinates, and $d \Omega^{2}=d \theta^{2}+\sin ^{2} \theta d \varphi^{2}$. The $\mathrm{U}(1)$ field is purely electric, with $A=V(r) d t$. A general spinors Ansatz compatible with the symmetries of the considered line element is [11]

$$
\Psi_{\epsilon}=\mathrm{e}^{-\mathrm{i} w t} \mathcal{R}_{\epsilon}(r) \otimes \Theta_{\epsilon}(\theta, \varphi),
$$


with $w$ the frequency and

$$
\begin{aligned}
& \mathcal{R}_{1}=-i \mathcal{R}_{2}=\left[\begin{array}{c}
\phi(r) \\
-i \bar{\phi}(r)
\end{array}\right], \\
& \Theta_{1}=\left[\begin{array}{c}
-\kappa \sin \frac{\theta}{2} \\
\cos \frac{\theta}{2}
\end{array}\right] e^{i \frac{\varphi}{2}}, \quad \Theta_{2}=\left[\begin{array}{c}
\kappa \cos \frac{\theta}{2} \\
\sin \frac{\theta}{2}
\end{array}\right] e^{-i \frac{\varphi}{2}},
\end{aligned}
$$

with $\kappa= \pm 1$. Also, assuming $r>0$, one considers the usual tetrad choice, with $e^{r}=\sqrt{g_{r r}} d r, e^{\theta}=\sqrt{g_{\Omega \Omega}} d \theta$, $e^{\varphi}=\sqrt{g_{\Omega \Omega}} \sin \theta d \varphi, e^{t}=\sqrt{-g_{t t}} d t$.

A useful parametrization in the numerics is $\phi=$ $|\phi| e^{i \alpha / 2}=e^{i \pi / 4} F-e^{-i \pi / 4} G$. Then the entire matter content of the model is encoded in the two real fermion functions $F(r), G(r)$, together with the electrostatic potential $V(r)$. This is essentially the framework used in 12] to construct (topologically trivial) particle-like solutions of the EDM system. In what follows we show that the system possess also traversable WH configurations [13].

An exact solution.- The resulting EDM equations can be solved analytically in the $q=0$ limit, the spinor fields being massless, with $w=0$. The solution has the metric and the $\mathrm{U}(1)$ potential

$d s^{2}=-\left(1-\frac{M}{r}\right)^{2} d t^{2}+\frac{d r^{2}}{\left(1-\frac{r_{0}}{r}\right)\left(1-\frac{Q_{e}^{2}}{r_{0} r}\right)}+r^{2} d \Omega^{2}$,

$V(r)=\frac{M}{Q_{e}} \sqrt{\left(1-\frac{r_{0}}{r}\right)\left(1-\frac{Q_{e}^{2}}{r_{0} r}\right)}$, with $M=\frac{2 Q_{e}^{2} r_{0}}{Q_{e}^{2}+r_{0}^{2}}$,

while the spinor functions are

$$
\begin{aligned}
& F(r)=c_{0}\left(\sqrt{1-\frac{Q_{e}^{2}}{r_{0} r}}-\kappa \sqrt{1-\frac{r_{0}}{r}}\right)^{2} / \sqrt{1-\frac{M}{r}}, \\
& G(r)=\frac{\kappa r_{0}}{32 c_{0}\left(Q_{e}^{2}+r_{0}^{2}\right)} \frac{\left(\sqrt{1-\frac{Q_{e}^{2}}{r_{0} r}}+\kappa \sqrt{1-\frac{r_{0}}{r}}\right)^{2}}{\sqrt{1-\frac{M}{r}}},
\end{aligned}
$$

with $c_{0} \neq 0$ an arbitrary constant. This describes a (regular) traversable WH solution, with $r_{0}$ the throat's radius and $Q_{e}<r_{0}$ the electric charge, while $M$ is the $\mathrm{ADM}$ mass (note that $Q_{e} / M>1$ ). The WH geometry is supported by the spinors contribution to the total energy-momentum tensor, being regular everywhere. As $Q_{e} \rightarrow r_{0}$, the extremal ReissnerNordström (RN) BH is approached, while $T_{\mu \nu}^{(D)} \rightarrow 0$.

Although this solution captures some basic properties of the general configurations below, it also possesses some undesirable features. In particular, the spinor wave function is not normalizable, since $|\phi|$ does not vanish as $r \rightarrow \infty$. However, the situation changes in a model with massive fermions, they becoming exponentially localized.
The general case.- The generic solutions have $\mu \neq 0, q \neq 0$ and are found numerically, by employing a metric ansatz which makes transparent the WH structure and simplifies the numerics [7]

$$
d s^{2}=-e^{2 \nu(r)} d t^{2}+f(r) d r^{2}+\left(r^{2}+r_{0}^{2}\right) d \Omega^{2},
$$

with $r_{0}>0$ the radius of the throat, which is located at $r=0$ (with $A_{T}=4 \pi r_{0}^{2}$ the throat area). The WH consists in two different regions $\Sigma_{ \pm}$of the same Universe. The 'up' region is found for $0<r<\infty$; there is also a 'down' region, with $-\infty<r<0$. However, in general the joining at $r=0$ of these regions is not 'smooth', with a discontinuity of the metric derivatives. This implies the presence of a thin mass shell structure at the throat, with a $\delta$-source added to the action (11) (e.g. the surface energy density is $\left.\epsilon_{T}=-4 \nu^{\prime}(0) / \sqrt{f(0)}\right)$. The condition for a 'smooth' geometry is $\nu^{\prime}(0)=0$.

Also, we shall consider the case of a symmetric WH, the geometry (6) and the energy-momentum tensor being invariant under the transformation $r \rightarrow-r$. The sign change of $r$ at the WH's throat reflects in a change of sign of the tetrad [16]. Then the matter functions transform as $V\left(r^{\prime}\right)=-V(r)$ and $\phi\left(r^{\prime}\right)=i \bar{\phi}(r)$ (with $r^{\prime}=-r>0$ ), while $\kappa \rightarrow-\kappa$ and $w \rightarrow-w$. As such, in what follows we shall report results mainly for the $r \geq 0$ region.

With this framework, the problem reduced to solving a system of four first order equations for $\{\nu, f, F, G\}$ and a second order equation for $V$ [18]. These equations are invariant under the transformation $w \rightarrow w+\beta, V \rightarrow V+\beta / q$ (with $\beta$ an arbitrary constant), which is fixed by imposing the electric potential to vanish at the throat.

The only global charges are the mass $M$ and the electric charge $Q_{e}$, which are read from the far field asymptotics. For the 'up' region, one finds $\nu \rightarrow$ $-M / r, f \rightarrow 1+2 M / r, V \rightarrow \Phi-Q_{e} / r$, (with $\Phi$ the electrostatic potential). The spinor functions decay as $e^{-\mu_{*} r} / r$, where $\mu_{*}=\sqrt{\mu^{2}-(w-\Phi / q)^{2}}$ (with the bound state condition $\left.\mu_{*}^{2} \geq 0\right)$.

An approximate solution can also be found close the throat, with the boundary conditions $\nu(0)=\nu_{0}$, $f(0)=f_{0}, F(0)=0, G(0)=G_{0}$ and $V(0)=0\left(\nu_{0}\right.$, $f_{0}, G_{0}$ being nonzero constants).

The WHs satisfy a Smarr law, the mass being the sum of an electrostatic term and a bulk contribution

$$
M=\Phi Q_{e}+M_{(B)},
$$

with

$M_{(B)}=4 \int_{0}^{\infty} d r\left(r^{2}+r_{0}^{2}\right)\left(\mu \sqrt{f} e^{\nu}\left(F^{2}-G^{2}\right)+q V|\phi|^{2} \sqrt{f}\right)$.

By integrating the Maxwell equations, one finds

$$
Q_{e}=2 q Q_{N}+Q_{T},
$$


where $Q_{N}$ is the Noether charge of a spinor (or number of particles)

$Q_{N}=\frac{1}{4 \pi} \int_{\Sigma_{+}} d^{3} x \sqrt{-g} j_{\epsilon}^{t}=4 \int_{0}^{\infty} d r \sqrt{f}\left(r^{2}+r_{0}^{2}\right)|\phi|^{2}$,

and $Q_{T}=V^{\prime}(0) e^{-\nu(0)} r_{0}^{2} / \sqrt{f(0)}$. Similar relations hold for the $r<0$ region, with mass, electric charge and Noether charge changing sign.

The equations of the model are invariant under the scaling transformation (the variables and quantities which are not specified remain invariant): $\left(r, r_{0}\right) \rightarrow \lambda\left(r, r_{0}\right),(F, G) \rightarrow(F, G) / \sqrt{\lambda},(\mu, q, w) \rightarrow$ $(\mu, q, w) / \lambda$, where $\lambda$ is a positive constant, while various quantities of interest transform as $\left(M, Q_{e}\right) \rightarrow$ $\lambda\left(M, Q_{e}\right),\left(Q_{N}, A_{H}\right) \rightarrow \lambda^{2}\left(Q_{N}, A_{H}\right)$. Only quantities which are invariant under this transformation (like $\left.M / Q_{e}\right)$ are relevant.

As with the solitons [12], 17], 20], this transformation is used to impose the one particle condition, $Q_{N}=1$, for each spinor in both 'up' or 'down' regions.

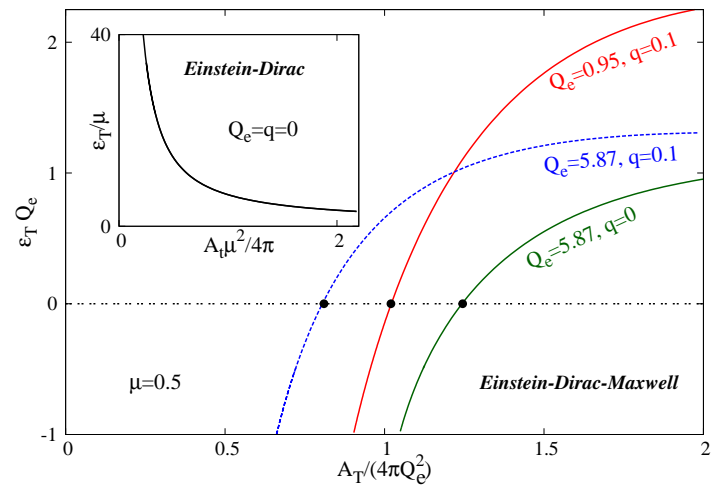

FIG. 1. The scaled thin layer energy density at the throat, $\epsilon_{T}$, is shown as a function of the scaled throat area $A_{T}$ for several sets of solutions at fixed frequencies.

The solutions.- We have solved the EDM for various values of the model's constants $(\mu, q)$. In particular, WH solutions exist also in the ED limit (i.e. $q=0$ and $V=0)$. However, as seen in the inlet of Fig. 1, those solutions have always $\nu^{\prime}(0) \neq 0$, and thus require the presence of extra-matter at the throat. The 'smooth' configurations necessarily possess a nonzero electric charge and have $\mu>0$ (although $q$ can vanish), the profile of a typical such configuration (marked with a star in Fig. 3). being displayed in Fig. 2.

In our approach, apart from $(q, \mu)$, the other input parameters are $\left\{Q_{e}, r_{0}, w\right\}$, all other quantities (e.g. $M$ and $Q_{N}$ ) being read from the numerical output. As shown in Fig. 1, our results indicate that for fixed
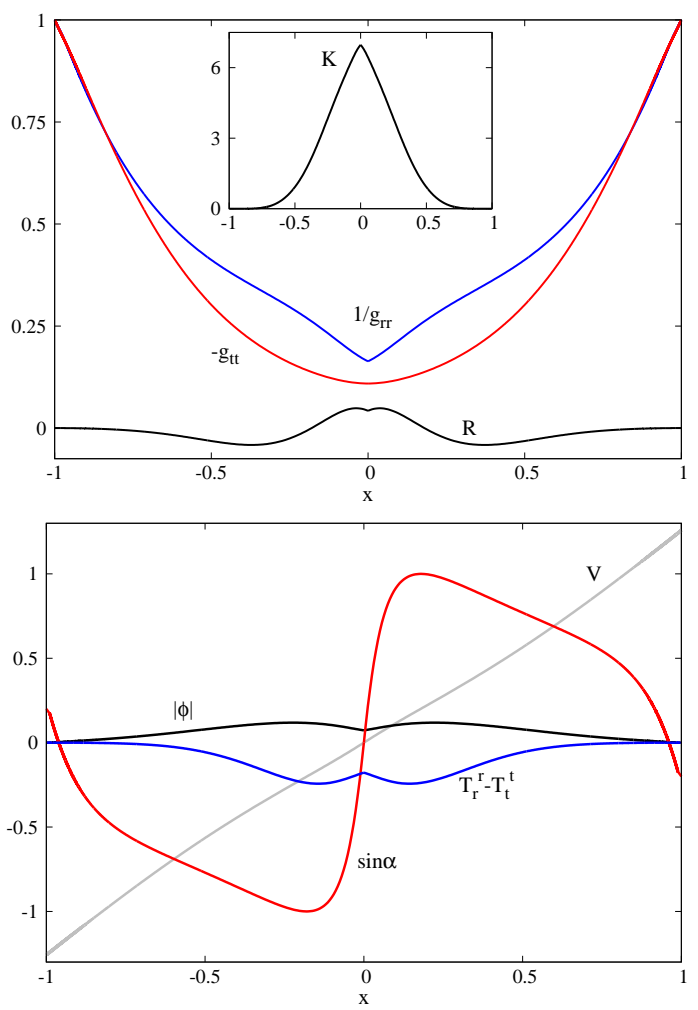

FIG. 2. The profile of a typical wormhole solution is displayed together with the Ricci and Kretschmann scalars (with $x=\frac{2}{\pi} \arctan \left(r / r_{0}\right)$ a compactified radial coordinate). The violation of the null energy condition is also shown.

electric charge and field frequency, a solution with no extra-matter at the throat exists for a unique value of the throat size [21].

As such, when varying $w$, a continuous set of 'smooth' solutions is found, the corresponding picture in terms of mass vs. throat area being shown in Fig. 3 (with the quantities given in units of the electric charge). A curve there interpolates between the extremal RN BH (in which limit $r_{0}$ becomes the horizon radius while the spinor fields vanish), and a critical configuration with $\mu_{*} \rightarrow 0$. This behaviour is generic, being found for all considered values of $(q, \mu)$. The set of all critical configurations forms the critical line. Although they still possesses a smooth geometry, their ADM mass is negative, a feature shared by a set of solutions close to them. Also, we have found that all solutions constructed so far have $Q_{e} / M>1$ and $q / \mu<1$.

A complementary picture is shown in the inset of Fig. 3, with the quantities given in Planck units (with the one particle condition imposed for each spinor). 


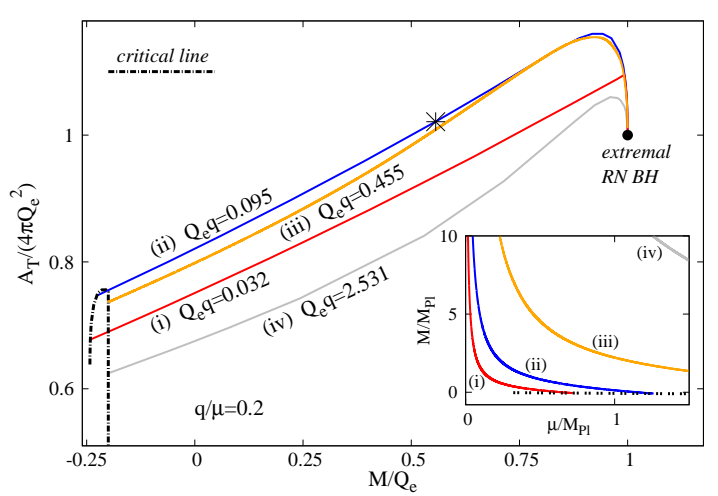

FIG. 3. The scaled area of the throat vs. the scaled ADM mass is shown for families of wormhole solutions. The curves starts from the extremal Reissner-Nordström black hole and end in a critical line where the spatial localization of the spinors is lost.

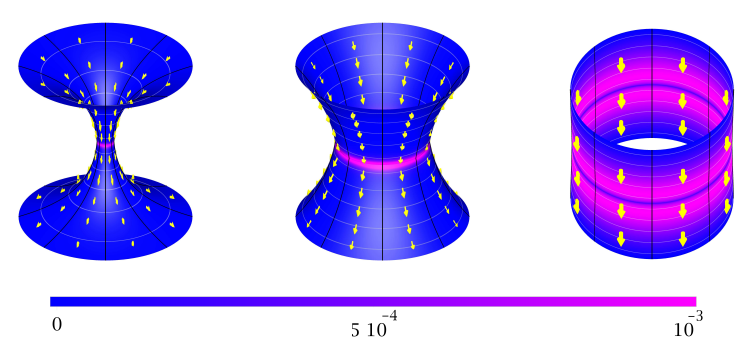

FIG. 4. A sequence of isometric embeddings, with a zoomed throat region, is shown for the wormhole solution in Fig. 2. The color map shows the absolute value of the Noether charge density.

When the mass $\mu$ of the spinors is made smaller and smaller, the solutions get arbitrarily close to extremal $\mathrm{RN}$ BHs, while the ADM mass appears to increase without bounds (note that, since the product $Q_{e} q$ is constant along the colored lines in Fig. 2, $Q_{e}$ and $q$ behaves in this limit as $M$ and $\mu$, respectively). On the other hand, the largest values found for $\mu$ are of order $10^{2} M_{P l}$, being approached at the critical line.

Essential for the existence of the WH solution is the violation of the null energy condition $T_{\mu \nu} n^{\mu} n^{\nu} \geq 0$, for any null vector field $n^{\mu}[5]$. The violation of this condition is displayed in Fig. 2, with $T_{r}^{r}-T_{t}^{t}<0$. The isometric embedding of the same WH solution is shown in Fig. 4, where the $\theta=\pi / 2$ plane is considered. The (absolute value of the) Noether charge density is also plotted there as a colour map (note that the maximal value of this quantity is approached outside the throat).
EDM WHs and entanglement.- In addition, the 'smooth', symmetric WHs have the Dirac fields at each side of the throat entangled in a particular way. Let us introduce two observers (Alice and Bob), which live in the asymptotically flat regions, where the solutions are approximately those of the flat space. Alice (at $r \rightarrow \infty$ ) sees the fermions in the state $\Psi_{\epsilon}^{A}=|\omega, \kappa\rangle$, while Bob (at $r \rightarrow-\infty$ ) sees the fermions in a state with opposite numbers, $\Psi_{\epsilon}^{B}=|-\omega,-\kappa\rangle$. The full asymptotic states will belong to the product of Alice and Bob Hilbert spaces, with $\Psi_{\epsilon}(|r| \rightarrow \infty)=\Psi_{\epsilon}^{A} \otimes \Psi_{\epsilon}^{B}=|\omega, \kappa\rangle \otimes|-\omega,-\kappa\rangle$. This corresponds to an entangled particle/antiparticle state of opposite chiralities [16]. The WHs cannot be 'smooth' unless the fermions are entangled in such a way. Also, since the electric flux smoothly enters the throat on one side and exits on the other, Bob observes the opposite electric flux and also measures opposite charges with respect to Alice (their frames being flipped).

Conclusions.- All known examples of traversable WHs with (classic) bosonic fields require some exotic matter and/or non-standard Lagrangians. However, the results in this work show that the situation changes for a fermionic matter content. WH solutions were found in the (standard) EDM theory, without introducing extra-matter in the bulk or at the throat, providing an explicit realization of Wheeler's idea of "electric charge without charge" 22. For the WHs to be 'smooth', the presence of a total electric charge is crucial, while to be traversable, the mass-charge ratio has to be smaller than one.

A semiclassical approach has been used, in which case the Dirac-Maxwell and Einstein equations are coupled, the fermionic matter being treated as a quantum wave function, a treatment which may provide a reasonable approximation under certain conditions [23]. However, we expect such configurations to exist as well in a more complete setting, with fully quantized matter fields 24], as suggested by the results in [27].

Also, although we considered a simple toy model with two localized fermions, this study can be extended to states with an arbitrary number of fermions, which would enhance the size of quantum effects, while retaining the simplifications offered by spherical symmetry [17], 28]. EDM WHs with a single spinor should also exist, possessing an intrinsic angular momentum 29]. Generalizations of such WH solutions for the full matter content of the Standard Model are also likely to exist.

Acknowledgements.- J.L.B.S. gratefully acknowledges support from DFG Project No. BL 1553, DFG Research Training Group 1620 Models of Grav- 
ity. The work of E.R. is supported by the Center for Research and Development in Mathematics and Applications (CIDMA) through the Portuguese Foundation for Science and Technology (FCT - Fundacao para a Ciência e a Tecnologia), references UIDB/04106/2020 and UIDP/04106/2020, and by national funds (OE), through FCT, I.P., in the scope of the framework contract foreseen in the numbers 4,5 and 6 of the article 23, of the Decree-Law 57/2016, of
August 29, changed by Law 57/2017, of July 19. We acknowledge support from the projects PTDC/FISOUT/28407/2017, CERN/FIS-PAR/0027/2019 and PTDC/FIS-AST/3041/2020. This work has further been supported by the European Union's Horizon 2020 research and innovation (RISE) programme H2020-MSCA-RISE-2017 Grant No. FunFiCO777740. The authors would like to acknowledge networking support by the COST Action CA16104.
[1] L. Flamm, Phys.Z. 17 (1916) 448.

[2] A. Einstein and N. Rosen, Phys. Rev. 48 (1935) 73.

[3] M. Wielgus, J. Horak, F. Vincent and M. Abramowicz, Phys. Rev. D 102, (2020) 084044.

[4] M. S. Morris and K. S. Thorne, Am. J. Phys. 56 (1988), 395-412

[5] M. Visser, Lorentzian wormholes: from Einstein to Hawking, (American Institute of Physics, Woodbury, 1995).

[6] H. G. Ellis, J. Math. Phys. 14, 104 (1973); K. A. Bronnikov, Acta Phys. Polon. B 4, 251 (1973); T. Kodama, Phys. Rev. D 18, 3529 (1978); C. Armendariz-Picon, Phys. Rev. D 65, 104010 (2002); H. Huang and J. Yang, Phys. Rev. D 100, 124063 (2019)

[7] P. Kanti, B. Kleihaus and J. Kunz, Phys. Rev. Lett. 107 (2011), 271101 arXiv:1108.3003 [gr-qc]].

[8] C. Barcelo and M. Visser, Class. Quant. Grav. 17 (2000), 3843-3864 arXiv:gr-qc/0003025 [gr-qc]].

[9] We use the notation and conventions in Ref. [10].

[10] S. R. Dolan and D. Dempsey, Class. Quant. Grav. 32 (2015) no.18, 184001 arXiv:1504.03190 [gr-qc]].

[11] J. L. Blazquez-Salcedo and C. Knoll, Eur. Phys. J. C 80 (2020) no.2, 174 arXiv:1910.03565 [gr-qc]].

[12] F. Finster, J. Smoller and S. T. Yau, Phys. Lett. A 259 (1999), 431-436 arXiv:gr-qc/9802012 [gr-qc]].

[13] As shown in Refs. [14], 15], the EDM solitons possess no $\mathrm{BH}$ generalizations.

[14] F. Finster, J. Smoller and S. T. Yau, Commun. Math. Phys. 205 (1999), 249-262 arXiv:gr-qc/9810048 [grqc]].

[15] F. Finster, J. A. Smoller and S. T. Yau, arXiv:gr-qc/9910030 [gr-qc]].

[16] M. Cariglia and G. W. Gibbons, arXiv:1806.05047 [gr-qc]].

[17] F. Finster, J. Smoller and S. T. Yau, Phys. Rev. D 59 (1999), 104020 arXiv:gr-qc/9801079 [gr-qc]].
[18] The EDM equations are solved by employing a solver [19] which uses a collocation method for boundaryvalue ODEs together with a damped Newton method of quasi-linearization. Typical meshes include around $10^{4}$ points, the relative accuracy of the solutions being around $10^{-10}$.

[19] U. Ascher, J. Christiansen and R. D. Russell, Math. Comput. 33 (1979) no.146, 659-679.

[20] C. A. R. Herdeiro, A. M. Pombo and E. Radu, Phys. Lett. B 773 (2017), 654-662 arXiv:1708.05674 [grqc]].

[21] As with solitons 12], WHs with excited Dirac spinors may exist as well, but here we consider fundamental solutions only. Also, all numerical results have $\kappa=1$ (for $r>0$ ), although a (qualitatively) similar picture has been found for $\kappa=-1$.

[22] J. A. Wheeler, Geometrodynamics (Academic, New York, 1962).

[23] C. Armendariz-Picon and P. B. Greene, Gen. Rel. Grav. 35 (2003), 1637-1658 arXiv:hep-th/0301129 [hep-th]].

[24] However, the inclusion of quantum corrections to the stress-energy tensor may affect the properties of the solutions [25, 26].

[25] L. E. Parker and D. Toms, "Quantum Field Theory in Curved Spacetime", Cambridge: Cambridge University Press (2009).

[26] P. B. Groves, P. R. Anderson and E. D. Carlson, Phys. Rev. D 66, 124017 (2002)

[27] J. Maldacena, A. Milekhin and F. Popov, arXiv:1807.04726 [hep-th]].

[28] P. Leith, E.D., C. A. Hooley, K. Horne and D. G. Dritschel, Phys. Rev. D 101 (2020) no.10, 106012 arXiv:2002.02747 [gr-qc]].

[29] C. Herdeiro, I. Perapechka, E. Radu and Y. Shnir, Phys. Lett. B 797 (2019), 134845 arXiv:1906.05386 [gr-qc]]. 